Effects of non-equilibrium particle distributions in deuterium-tritium burning

D. Michta, F. Graziani, J. Pruet, T. Luu

August 19, 2009

Physics of Plasmas 
This document was prepared as an account of work sponsored by an agency of the United States government. Neither the United States government nor Lawrence Livermore National Security, LLC, nor any of their employees makes any warranty, expressed or implied, or assumes any legal liability or responsibility for the accuracy, completeness, or usefulness of any information, apparatus, product, or process disclosed, or represents that its use would not infringe privately owned rights. Reference herein to any specific commercial product, process, or service by trade name, trademark, manufacturer, or otherwise does not necessarily constitute or imply its endorsement, recommendation, or favoring by the United States government or Lawrence Livermore National Security, LLC. The views and opinions of authors expressed herein do not necessarily state or reflect those of the United States government or Lawrence Livermore National Security, LLC, and shall not be used for advertising or product endorsement purposes. 


\title{
Effects of non-equilibrium particle distributions in deuterium-tritium burning
}

\author{
David Michta* \\ University of California, Berkeley \\ Frank Graziani, ${ }^{\dagger}$ Jason Pruet, ${ }^{\ddagger}$ and Thomas Luu ${ }^{\S}$ \\ Lawrence Livermore National Laboratory
}

(Dated: August 18, 2009)

\begin{abstract}
We investigate the effects of non-equilibrium particle distributions resulting from rapid deuterium-tritium burning in plasmas using a Fokker-Planck code that incorporates small-angle Coulomb scattering, Brehmsstrahlung, Compton scattering, and thermal-nuclear burning. We find that in inertial confinement fusion environments, deviations away from Maxwellian distributions for either deuterium or tritium ions are small and result in $1 \%$ changes in the energy production rates. The deuterium and tritium effective temperatures are not equal, but differ by only about $2.5 \%$ near the time of peak burn rate. Simulations with high Z (Xe) dopants show that the dopant temperature closely tracks that of the fuel. On the other hand, fusion product ion distributions are highly non-Maxwellian, and careful treatments of energy-exchange between these ions and other particles is important for determining burn rates.
\end{abstract}

\footnotetext{
*dmichta@berkeley.edu

†graziani1@llnl.gov

${ }^{\ddagger}$ pruet1@llnl.gov

$\S_{\text {tluu@llnl.gov }}$
} 


\section{INTRODUCTION}

Non-equilibrium effects play an important role in inertial confinement fusion plasmas $[1,2]$. Timescales characterizing the themodynamic evolution of the light ion fuel are typically comparable to or shorter than those governing important equilibration processes. The dominant effect relates to ions falling out of equilibrium with electrons, and charged particles out of equilibrium with photons. This results in a disproportionately large fraction of the energy in the ions, and a concomittantly larger fusion rate [2]. Recently large-scale molecular dynamics simulations have been used by several groups to develop an improved understanding of the coupling coefficients governing the energy exchange rates between electrons and ions within plasmas $[3,4]$.

Here we investigate a class of more subtle non-equilibrium effects relating to equilibration within a single particle species and across heavy ion species. Though a more detailed description is given below, the qualitative motivation is the observation that fusion preferentially involves particles within the 'Gamow Peak' that accounts for the competition between the rise of the cross section and decrease of the distribution function with increasing energy. This raises the possibility that depletion of ions within this peak leads to a non-equilibrium distribution. Though this would not have much effect on the overall energetics, it could change burn rates. We also examine the equilibration between ion species, and in particular investigate the role of high $\mathrm{Z}$ dopants.

To study details of thermalization associated with the particle distribution function, one must go beyond the traditional Spitzer [5] model for bulk equilibration. We have developed a numerical algorithm of the Fokker-Planck equations that includes the physical effects of Coulomb scattering, Compton scattering, Brehmsstrahlung and inverse Brehmsstrahlung, and thermal-nuclear burning. Here the basic assumption is that the relaxation times of certain plasma processes are large compared to the intrinsic time scales of the problem (e.g. scattering events). As long as relative changes in plasma quantities remain small at each timestep (which we can control), the Fokker-Planck equations remain valid. Thus the entire time-evolution of plasma quantities (such as ion distributions) can be ascertained.

In the following section (II) we give a cursory explanation of our Fokker-Planck code, citing standard references for more detailed derivations. We enumerate the different types of physics phenomena included in the code and describe the coupling of therno-nuclear fusion 
with the Fokker-Planck formalism. We also show benchmark results of our code wherein we compare with known published results, thereby validating our numerical studies. In sect. III we show the results of various fusion simulations. We present results for systems in which D-T fusion is the primary reaction fuel. We compare our results to runs in which only equilibrium distributions are used, thereby quantifying the effects of any non-equilibrium processes. We also show how dopants, such as Xenon, quench the burn profile in our simulations. We conclude in sect. V.

\section{DESCRIPTION OF FOKKER-PLANCK CODE}

In what follows we give a cursory review of the Fokker-Planck routine and the various physics phenomena included in the algorithm. For more thorough discussions and derivations the reader is referred to refs. [6-9] and references within.

\section{A. Physics implemented in Fokker-Planck code}

The Fokker-Planck equation describes the time evolution of a particle velocity distribution in terms of its drift and diffusion in velocity space. This approach is valid where small momentum transfers or small-angle collisions are the dominant transport mechanism, such as in weakly coupled plasmas. The Fokker-Planck equation is

$$
\frac{\partial f}{\partial t}=\frac{C}{v^{2}} \frac{\partial}{\partial v}\left(F f+D \frac{\partial f}{\partial v}\right)
$$

with $\mathrm{F}$ the friction and $\mathrm{D}$ the diffusion coefficient due to all colliding species. The FokkerPlanck collision coefficients are moments of the colliding species' distributions, given by

$$
\begin{aligned}
F_{i}(v) & =\frac{m}{m_{i}} \int_{0}^{v} d u u^{2} f_{i}(u) \\
D_{i}(v) & =\frac{1}{3 v} \int_{0}^{v} d u u^{4} f_{i}(u)+\frac{v^{2}}{3} \int_{v}^{\infty} d u u f_{i}(u) .
\end{aligned}
$$

The coupling coefficient is given by

$$
C_{i}=\frac{4 \pi Z^{2} Z_{i}^{2} e^{4}}{m^{2}} \ln \Lambda
$$

where the coulomb logarithm between colliding species is defined as

$$
\ln \Lambda=\frac{1}{2} \ln \frac{r_{\text {ion }}^{2}+\lambda_{d}^{2}}{r_{\text {closest }}^{2}+\lambda_{\text {deBroglie }}^{2}},
$$


where $r_{i o n}$ is the charge-radius of the ion, $r_{\text {closest }}$ is the radius of closest approach, $\lambda_{\text {deBroglie }}$ is the thermal de Broglie wavelength of the colliding particles, and $\lambda_{d}$ is the Debye length. In general $\ln \Lambda$ takes on various forms depending on the physics approximations employed (see [10] and references within for a discussion of the various forms of $\ln \Lambda$ ).

Each particle species' Fokker-Planck equation is simply the sum of collision contributions from all species, so that the friction coefficient is $C F=\sum_{i} C_{i} F_{i}$ and similarly for the diffusion term. Number density is defined as $N \equiv 4 \pi \int d v v^{2} f(v)$.

The numerical utility of the Fokker-Planck equation becomes apparent after descretizing eq. 1, as the first and second derivatives become implicit difference equations related to nearest neighbor grid points. This sets up a tri-diagonal system of equations that can be solved efficiently in linear time. We enforce particle number conservation by using the generalized current discretization scheme given by Chang and Cooper [8]. This scheme has the added benefit that it ensures proper equilibration of distribution functions. Energy conservation is enforced by proper discretization of implicit collision coeficients as derived in $[11]$.

The radiation energy spectrum is also evolved in time through the processes of Compton scattering/inverse Compton scattering and Bremsstrahlung/inverse Bremsstrahlung. The photons couple to the plasma via the electrons. Since electron self-collision times are orders of magnitude shorter than those of ion-ion and electron-ion collisions, the electron distribution is assumed Maxwellian at all times. Thus the the electron temperature is sufficient to compute the radiation spectrum change, and the resulting energy difference is explicitly compensated in a re-Maxwellianized electron distribution.

Compton Scattering and inverse Compton scattering are modeled with the Kompaneets equation [12], a Fokker-Planck equation of the radiation energy spectrum. The Kompaneets equation is $[8,12]$

$$
\frac{\partial f}{\partial t}=\frac{n_{e} \sigma_{0}}{k^{2}} \frac{\partial}{\partial k}\left[k^{4}\left(T_{e} \frac{\partial f}{\partial k}+f+f^{2}\right)\right],
$$

where $k \equiv h \nu$ is the photon energy and $f(k, t) \equiv\left(\frac{h c}{2}\right)^{3} f_{p}(k, t)$ with $f_{p}$ being the photon distribution. The Kompaneets equation is solved analogously to the particle Fokker-Planck equations, where conservation of photon number and stable approach to the equilibrium Bose-Einstein distribution are ensured.

Bremsstrahlung and inverse Bremsstrahlung radiation are an important mechanism for plasma cooling and are implemented in the code. Radiation absorption and emission terms 
TABLE I: Fusion reactions included in study

\begin{tabular}{l}
\hline \hline Fusion reactions \\
\hline${ }^{2} \mathrm{H}+{ }^{2} \mathrm{H} \rightarrow{ }^{1} \mathrm{H}+{ }^{3} \mathrm{He}$ \\
${ }^{2} \mathrm{H}+{ }^{2} \mathrm{H} \rightarrow \mathrm{n}+{ }^{3} \mathrm{H}$ \\
${ }^{2} \mathrm{H}+{ }^{3} \mathrm{H} \rightarrow \mathrm{n}+{ }^{4} \mathrm{He}$ \\
${ }^{3} \mathrm{H}+{ }^{3} \mathrm{H} \rightarrow \mathrm{n}+\mathrm{n}+{ }^{4} \mathrm{He}$ \\
\hline \hline
\end{tabular}

for a Maxwellian electron distribution are taken from [13]. The power emitted due to electron-ion collisions per photon frequency range is given as

$$
\frac{d W}{d V d t d \nu}=\frac{2^{5} \pi e^{6}}{3 m c^{3}}\left(\frac{2 \pi}{3 k m}\right)^{1 / 2} T^{-1 / 2} Z^{2} n_{e} n_{i} e^{\frac{-h \nu}{k T_{e}}} \bar{g}_{f f}
$$

and the power absorbed by the electrons per photon frequency range is given as

$$
\frac{d W}{d V d t d \nu}=4 \pi \frac{4 e^{6}}{3 m h c}\left(\frac{2 \pi}{3 k m}\right)^{1 / 2} T_{e}^{-1 / 2} Z^{2} n_{e} n_{i}\left(1-e^{\frac{-h \nu}{k T_{e}}}\right) \bar{g}_{f f} B_{\nu}(T)
$$

The 'free-free' gaunt factor is denoted $\bar{g}_{f f} . B_{\nu}(T)$ typically represents the Planck function, however here the nonequilibrium photon intensity distribution is used. These quantities are implemented as source and implicit drain terms to be solved in the photon distribution tridiagonal system.

\section{B. Including thermo-nuclear fusion}

The thermonuclear fusion of ions and the production of daughter nuclei has been coupled to the Fokker-Planck code. A total of four fusion processes have been included and are enumerated in tab. I.

The rate at which species $i$ at a velocity $v$ is depleted (per unit volume) due to fusion with species $j$ is computed as a simple integral over the colliding species' distribution functions, their relative velocity, and their fusion cross-section $\sigma_{i j}$,

$$
\frac{d f_{i}(v)}{d t}=f_{i}(v) \int d \overrightarrow{v_{j}} f_{j}\left(v_{j}\right)\left|\overrightarrow{v_{i}}-\overrightarrow{v_{j}}\right| \sigma_{i j}\left(\left|\overrightarrow{v_{i}}-\overrightarrow{v_{j}}\right|\right) .
$$

Integrating this rate over all velocities for species $i$ gives the total fusion rate $R_{i j}$ per unit volume. 
For reactions with two fusion products $k$ and $l$, the non-relativistic outgoing energy distribution for species $k$, normalized to unity, is given by

$$
S_{k}(E)=\frac{n_{i} n_{j}}{R_{i j}} \frac{\sqrt{2}\left(m_{i}+m_{j}\right)^{3 / 2}}{\pi k T^{2} \sqrt{m_{i} m_{j} m_{k} m_{l}}} \int \frac{E_{r} \sigma_{i j}\left(E_{r}\right) e^{-E_{r} / k T}}{\sqrt{E_{r}+Q}}\left(e^{-\alpha_{k-}\left(E, E_{r}\right)}-e^{-\alpha_{k+}\left(E, E_{r}\right)}\right),
$$

where

$$
\begin{aligned}
\alpha_{k \pm}\left(E, E_{r}\right) & =\frac{m_{i}+m_{j}}{m_{k} k T}\left(\sqrt{E} \pm \sqrt{\frac{m_{l}}{m_{i}+m_{j}}\left(E_{r}+Q\right)}\right)^{2} \\
Q & =m_{i}+m_{j}-m_{k}-m_{l}
\end{aligned}
$$

Here $E_{r}$ is the relative energy of the fusing particles $i$ and $j$ and $n_{i}$ and $n_{j}$ are their respective number densities. Equation 10 is derived from its relativistic counterpart given in [14]. It is straightforward to convert eq. 10 into a velocity distribution to be used in our Fokker-Planck algorithm. These annihilation and creation rates, as well as the annihilation and creation distributions, are included as source and drain terms in the Fokker-Planck tridiagonal system.

\section{Benchmark tests}

We have benchmarked our algorithm to various known physical processes. In conditions involving only small-angle Coulomb scattering (all other physical processes 'turned off') and two species $i$ and $j$ intially at different temperatures, the evolution of the temperatures of these species is governed by Spitzer's equipartition theory [5]

$$
\frac{d T_{i}}{d t}=\frac{T_{j}-T_{i}}{\tau_{i j}}
$$

where

$$
\tau_{i j}=\frac{3 m_{i} m_{j} k^{3 / 2}}{8(2 \pi)^{1 / 2} n_{f} Z_{i}^{2} Z_{j}^{2} e^{4} \ln \Lambda}\left(\frac{T_{i}}{m_{i}}+\frac{T_{j}}{m_{j}}\right)^{3 / 2} .
$$

Our Fokker-Planck code is shown to agree well with Spitzer's theory. Also, we have reproduced the results given in [7] related to deuteron-electron relaxation.

In conditions where Compton scattering is the dominant equilibration process, we have verified that particle distributions and temperatures approach the correct equilibrium solution, i.e. ions approach Maxwellian distributions and photons approach Planckian distributions. Our comptonization rates match those obtained by other other Kompaneets solvers, such as those given by Shirk [15]. 
Finally, Bremsstrahlung and inverse Bremsstrahlung radiation have been verified against the expected equilibrium result. Calculated photon fugacities consistently approach 1, indicating equilibration of the photon field to a blackbody distribution. Total power of emitted Bremsstrahlung radiation is [13]

$$
\frac{d W}{d t d V}=\frac{2^{5} \pi e^{6}}{3 h m c^{3}}\left(\frac{2 \pi k T}{3 m}\right)^{1 / 2} Z^{2} n_{e} n_{i} \bar{g}_{f f}
$$

Calculated emission power from our Fokker-Planck code compares well with the above analytic expression.

\section{RESULTS}

In the runs shown below, initial conditions have equimolar concentrations of deuterium and tritium and zero concentrations of fusion products. All ions are held at some specified initial temperature (at time $t=0$ ). In addition, electrons and a photon blackbody background are both included at the same initial temperature. The photon density is set by the temperature and blackbody distribution, whereas the electron density is chosen to ensure electrical neutrality (the deuterons and tritons are assumed fully ionized). As the plasma undergoes burning, the number densities of fusion products grow accordingly. We do not track any fusion neutrons.

\section{A. Deuterium-Tritium fusion}

In fig. 1 we show a typical run in which D-T is the primary fuel. Initial conditions consist of equimolar amounts of D-T corresponding to $1000 \mathrm{~g} / \mathrm{cm}^{3}$ held at initial temperature $\mathrm{kT}=2$ $\mathrm{keV}$. In fig. 2 we show the effective temperature $T_{\text {eff }}[13]$, expressed in units of $\mathrm{keV}$, of the photon distribution.

We can characterize the burn profile shown in fig. 1 into three main regions. There is an initial 'cooking' phase where all particles have similar temperatures and little is happening. Here the fusion rate is small and the number density of alphas grows slowly, as shown in fig. 3. Alphas are produced with roughly 3.6 MeV of kinetic energy. They subsequently down scatter and impart their energy onto deuterium, tritium, and electrons. The electrons then impart their energy onto the background radiation. This initial alpha production, though 


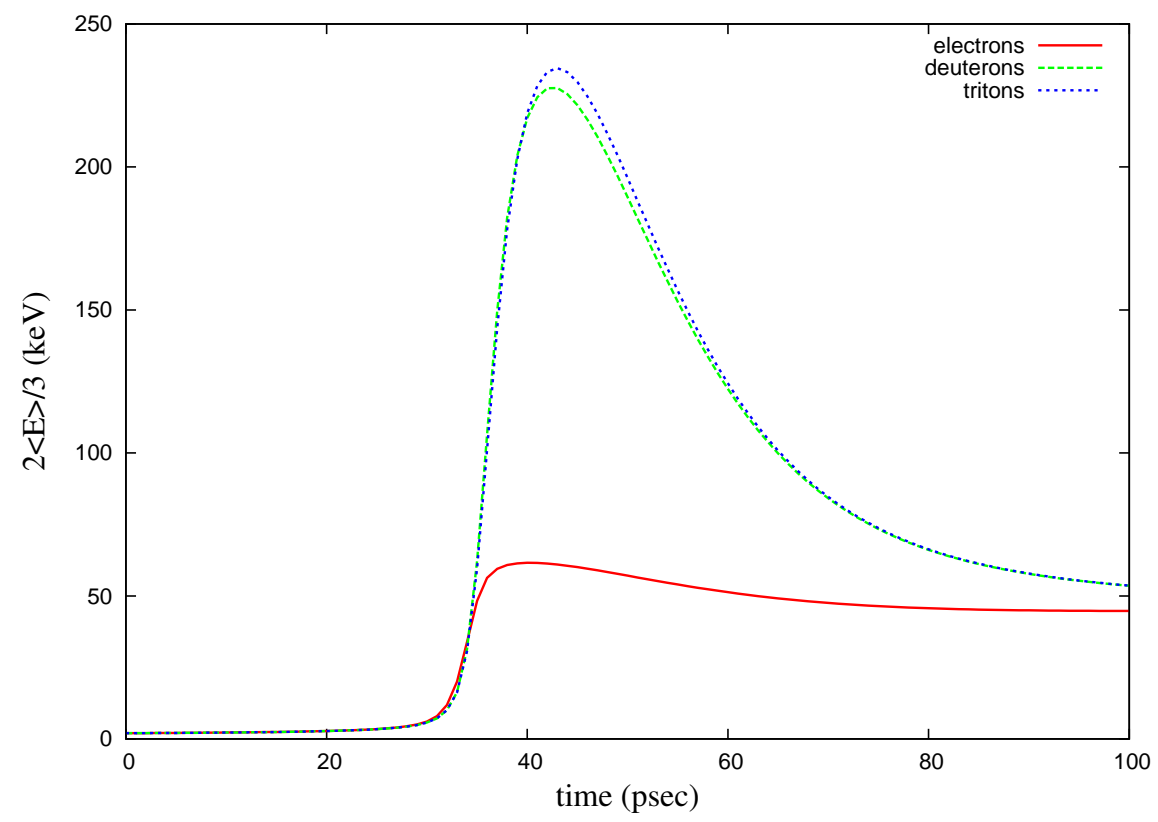

FIG. 1: Typical burn profile for plasma with deuterium-tritium as the primary reaction fuel. Here $<E>$ represents the mean energy of the particle distributions, and what is plotted on the ordinate is $2 / 3$ this value. If the particles were in equilibrium, this would correspond to the temperature of the particles. The deuterium, tritium, electron, and photon temperatures (not shown in this figure) were initially held at $k T=2 k e V$, with equimolar amounts of deuterium and tritium corresponding to $1000 \mathrm{~g} / \mathrm{cm}^{3}$.

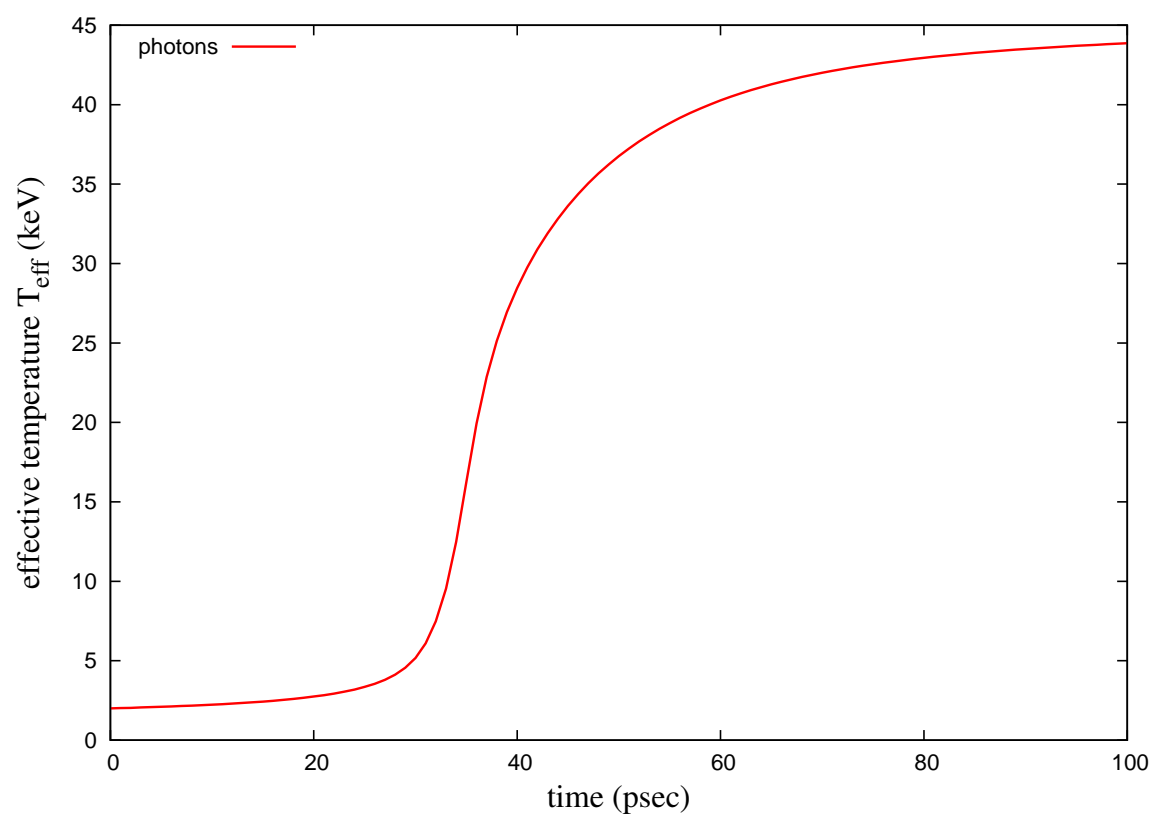

FIG. 2: Effective temperature profile of photon distribution as a function of time. 


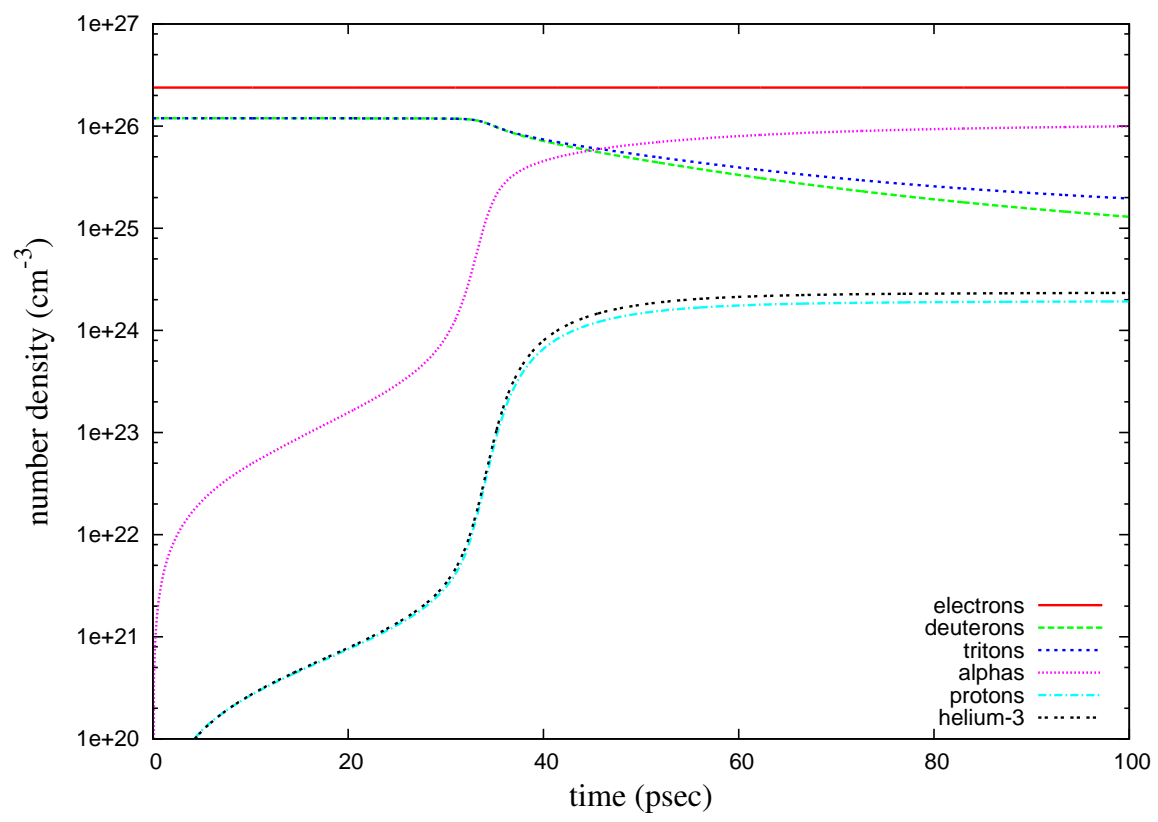

FIG. 3: Number densities of the various particle species as a function of time.

small, heats the ions and increases the fusion rate. This positive feedback ultimately leads to the system undergoing runaway burn, characterizing the second region of the profile. Here the deuterium and tritium 'light', and their respective temperatures grow faster than the electron and photon temperatures; the system is no longer in equilibrium with all particle species. During this phase the energies are high enough that fusion reactions other than D-T start to occur. Figure 4 shows the reaction rates for various fusion processes as a function of time. The burn cannot sustain itself since the deuterium/tritium fuel depletes and their number densities decrease to such a point that fusion rates are negligible. The system thus 'cools' to equilibrium in the third region with a timescale dictated by Coulomb energy exchange rates and Brehmsstrahlung.

Up to this point we have avoided the use of 'temperature' for a statistical measure of ion properties. It is not clear that any of the particle species are in equilibrium with each other, especially during the lighting phase. We note that if the different species are in equilibrium (i.e. have Maxwell-Boltzmann distributions), then $k T=2<E>/ 3$ and the ordinates of fig 1 can be replaced with temperature. This is always true for electrons since their small mass gives them short equilibration times ( $\tau_{\text {electron }} \ll$ picosecond). Therefore they remain in equilibrium throughout the entire run. For photons, if they are in equilibrium and thus characterized by a Planckian distribution, the effective temperature is the same as the thermal blackbody temperature, $T_{\text {eff }}=T$. As we show in the next section, the deuterium 


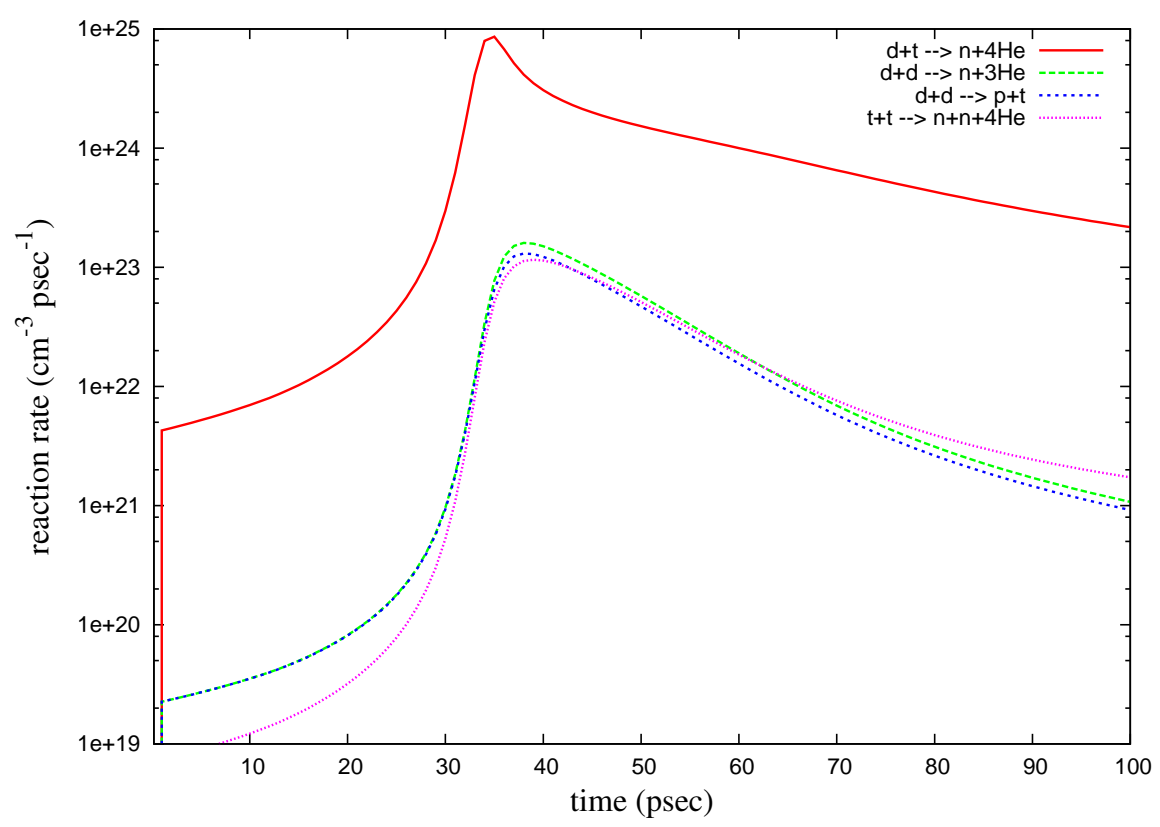

FIG. 4: Reaction rates for various fusion processes as a function of time.

and tritium distributions are never far from equilibrium, even during the lighting stage. This is not the case, however, for photons and fusion products (e.g. alphas, protons, helium-3). We will show examples of these cases later.

\section{Deuterium and tritium distributions}

In fig. 5 we show the energy distributions of the deuterons and tritons, normalized to their respective number densities, at various times during the burn simulation. Note the log-log scale of the plots. In panel (a) of fig. 5 we also show a kT=2 keV Maxwell-Boltzmann distribution normalized to $10^{25}$ particles per $\mathrm{cm}^{3}$ for comparison. For the triton distributions there is a clear non-equilibrium component that comes from the fusion reaction

$$
d+d \rightarrow p+t
$$

This reaction produces tritons with approximately $1 \mathrm{MeV}$ of kinetic energy. For the deuteron distributions it is difficult to see from fig. 5 whether any non-equilibrium contributions are present. Both deuterium and tritium have the bulk of their distributions in equilibrium, especially at low energies below $100 \mathrm{keV}$. In this region we fit a Maxwellian distribution using a simple two-point formula: Given two coordinates of the distribution function, $\left(\mathrm{E}_{1}, \mathrm{f}\left(\mathrm{E}_{1}\right)\right)$ and $\left(\mathrm{E}_{2}, \mathrm{f}\left(\mathrm{E}_{2}\right)\right)$, we can extract the thermal bulk temperature $k T_{b g}$ and the number density 


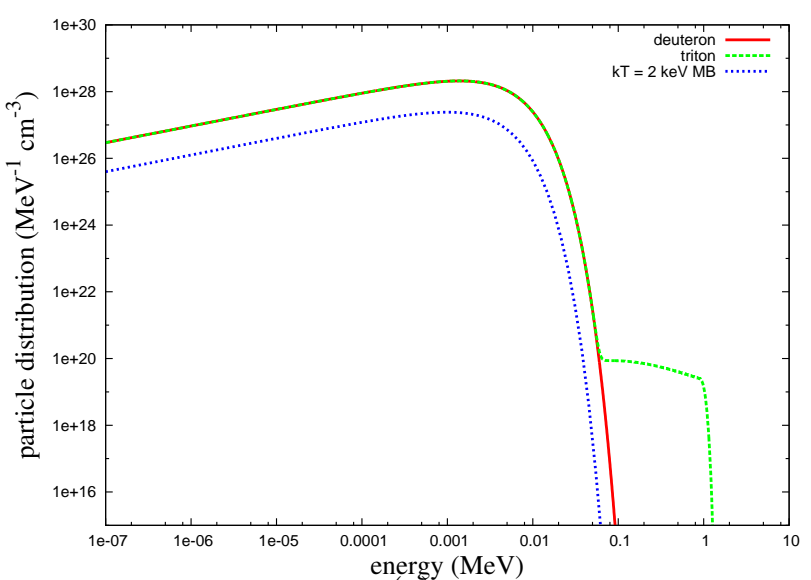

(a)

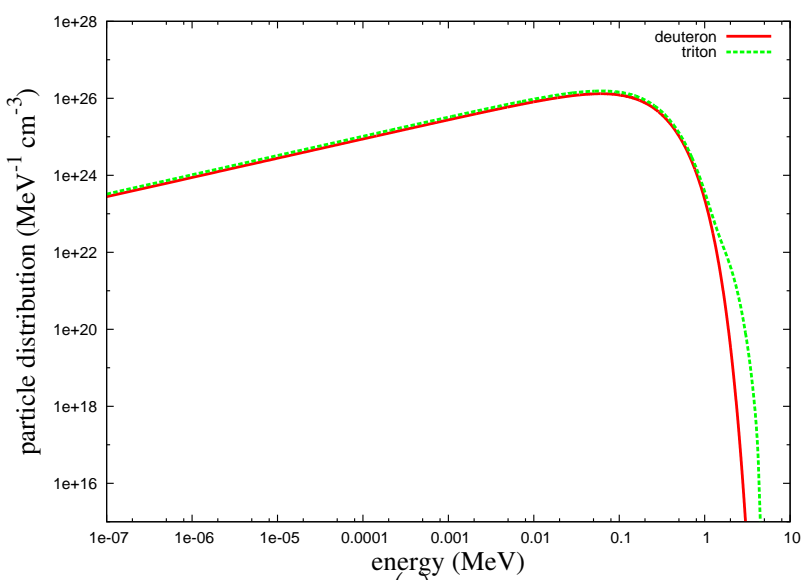

(c)

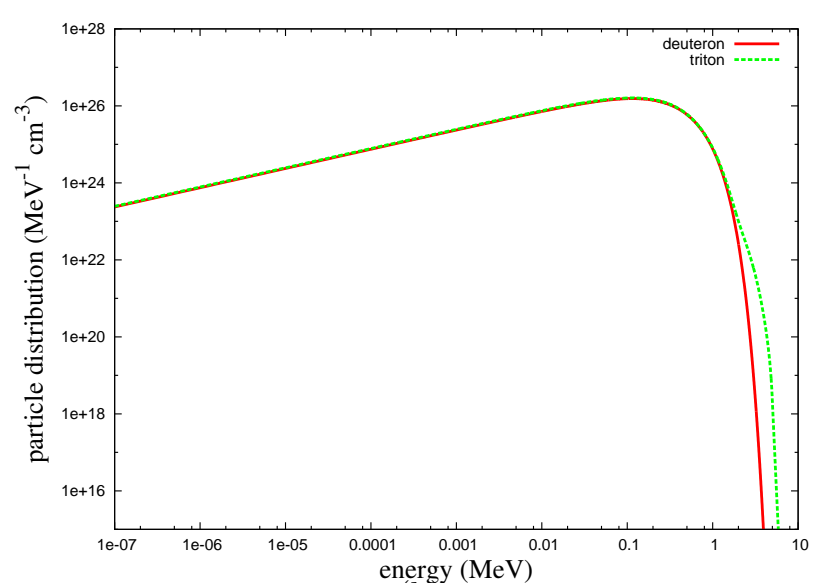

(b)

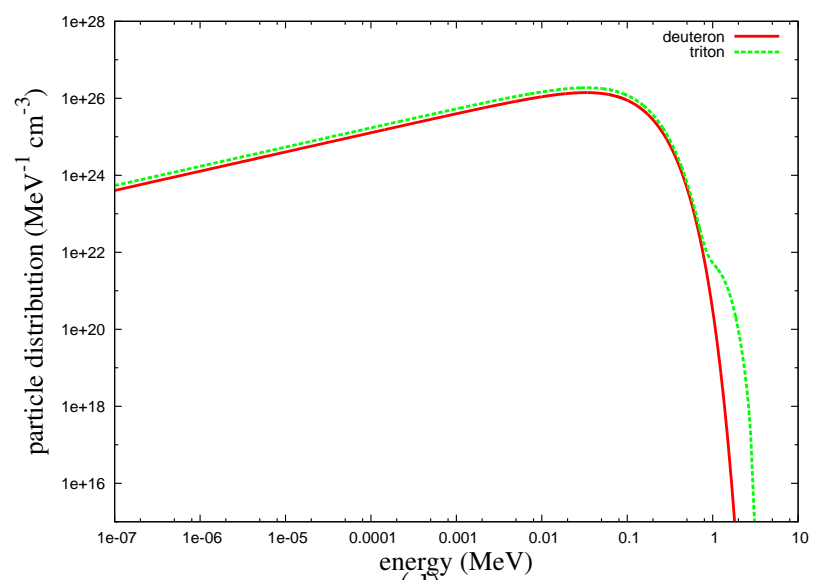

(d)

FIG. 5: Deuteron and triton energy distributions at various times during the burn calculation. Panel (a) shows distributions at $\mathrm{t}=20$ psecs, (b) $\mathrm{t}=40$ psecs, (c) $\mathrm{t}=60$ psecs, and (d) $\mathrm{t}=80$ psecs. For comparison panel (a) also shows a sample Maxwellian distribution with temperature $k T=2$ $\mathrm{keV}$ and normalized to $10^{25}$ particles per $\mathrm{cm}^{3}$.

of equilibrium particles $N_{e q}$ using

$$
\begin{aligned}
k T_{b g} & =\left(E_{2}-E_{1}\right) \ln \left(\frac{f\left(E_{1}\right) \sqrt{E_{2}}}{f\left(E_{2}\right) \sqrt{E_{1}}}\right) \\
N_{e q} & =\frac{\sqrt{\pi}\left(k T_{b g}\right)^{3 / 2}}{2 \sqrt{E_{1}}} f\left(E_{1}\right) e^{E_{1} / k T_{b g}} .
\end{aligned}
$$

When extracting these terms, we choose $\mathrm{E}_{1}$ and $\mathrm{E}_{2}$ well below $100 \mathrm{keV}$, typically in the $\mathrm{keV}$ or less range. We note that if $\mathrm{f}(\mathrm{E})$ is exactly Maxwellian, then Eqs. 16 and 17 are exact. That is, $k T_{b g}$ corresponds to the exact thermal temperature and $N_{e q}$ corresponds to the exact number density. Given the total number density of a particular ion, $N_{\text {tot }}$, and the density of particles of that same ion in equilibrium $N_{e q}$, the density of non-equilibrium 


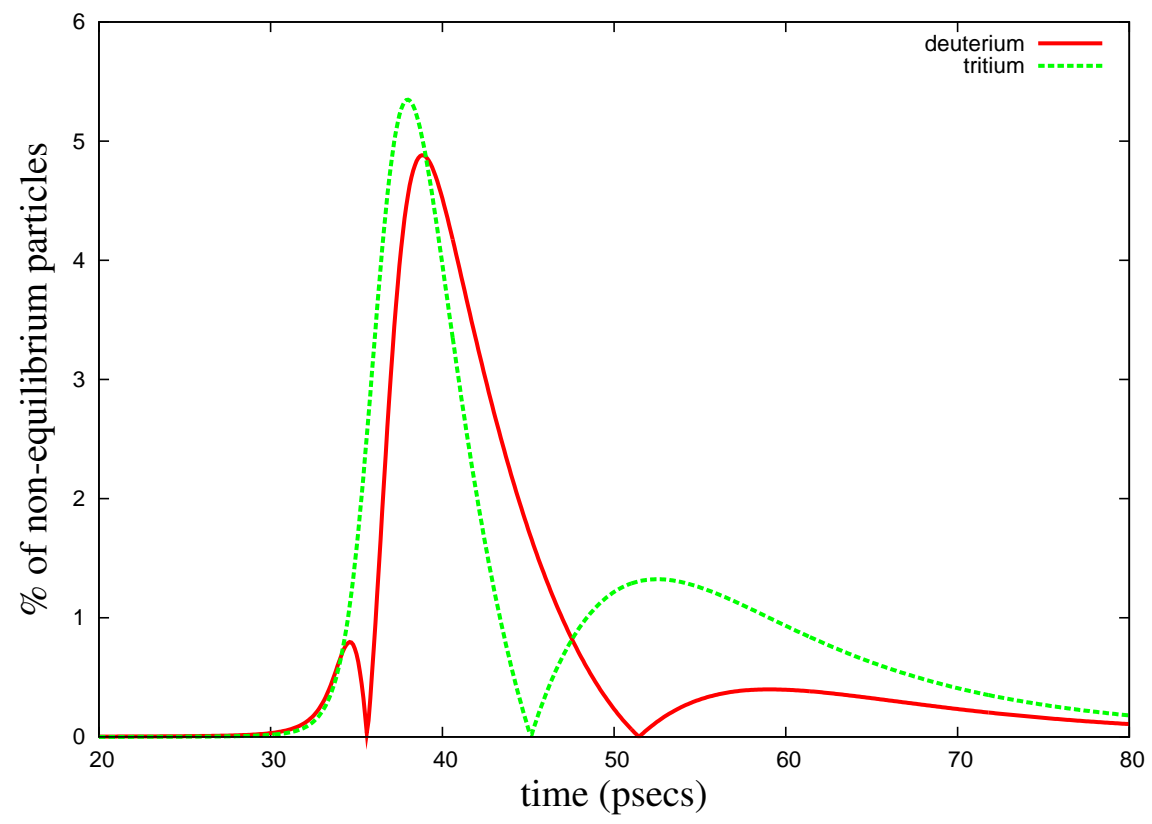

FIG. 6: Percentage of deterium/tritium not in equilibrium as a function of time.

particles is given by the difference of these quantities. We show in fig. 6 the percentage of particles not in equilibrium calculated in this fashion as a function of time for deuterium and tritium. The percentages are largest during and after the lighting stage, growing no larger than $5.5 \%$.

Another figure of merit that details the extent in which any particle distribution $f(E)$ (other than photon) deviates from a Maxwellian distribution is the excess kurtosis,

$$
K_{e x}=\frac{\frac{1}{N_{t o t}} \int d E E^{2} f(E)}{\left(\frac{1}{N_{t o t}} \int d E E f(E)\right)^{2}}-3,
$$

where $N_{t o t}=\int d E f(E)$. For a Maxwellian distribution, $K_{e x}=0$. A positive excess indicates a distribution that is more weighted in the tail, whereas a negative excess indicates a distribution weighted more at low energies. In fig. 7 we show the excess kurtosis of the deuterium and tritium ion distributions for the run described earlier. Initially, during the 'cooking' phase, the excess kurtosis is zero and the deuteron/triton distributions are Maxwellian. As the plasma ignites the kurtosis goes negative, indicating that the low-energy region of the distributions is enhanced relative to the high-energy region. This is to be expected since fusion predominantly occurs in the high-energy region about the Gamow peak, thereby decreasing the distributions in this region. During the cooling stage the excess becomes positive. Here fusion has basically ceased and the remaining high-energy fusion products 


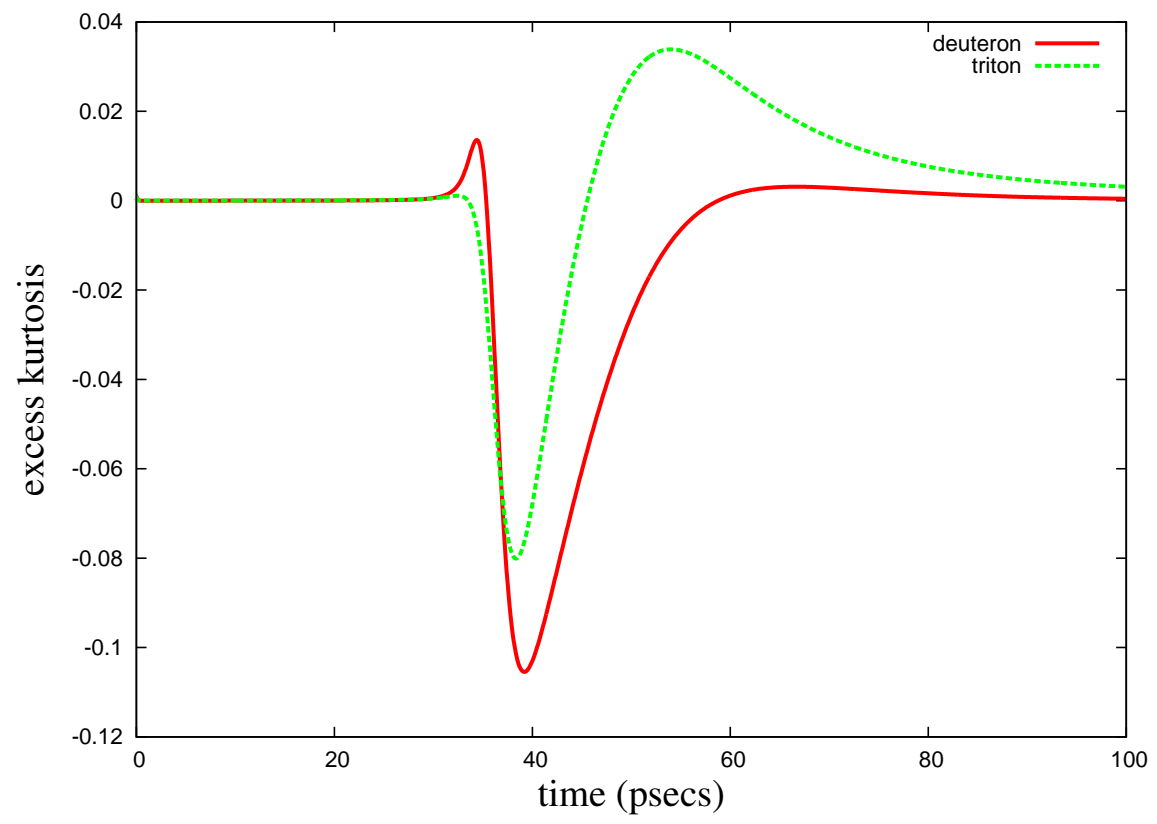

FIG. 7: Excess kurtosis of deuterium and triton distributions as defined in eq. 18.

impart their energy onto deuterium and tritium (and electrons), enhancing the tail end of their distributions.

Figures 5-7 indicate deviations from equilibrium for the fusion ions. Do these nonequilibrium distributions drive large effects in our burn evolution? To answer this question, we repeat our simulation but with a constraint that (only) the deuterons and tritons remain in equilibrium after every timestep. We enforce this constraint by assigning Maxwellian distributions to these ions with temperatures derived from the Fokker-Planck mean energies, $\langle E\rangle=\frac{3}{2} k T$. Figure 8 shows the comparison of deuteron and triton mean energies calculated in this manner with mean energies calculated in the original Fokker-Planck simulation, showing little difference. Figure 9 shows the percent difference between deuteriumtriton fusion rates calculated using the Fokker-Planck simulation and one calculated with Maxwell-Boltzmann enforcement. Largest differences occur again around peak burn, but are limited to at most $1.3 \%$. These results indicate that effects due to any non-equilibrium distributions of the deuterons and tritons play a small role in the evolution of the burning plasma[16].

At peak burn the temperatures of the triton and deuteron begin to differ, as shown in fig. 10. Tritons obtain a larger peak temperature due to more favorable energy exchange rates with the alphas (there is a smaller mass difference between tritons and alphas as opposed to deuterons and alphas). This suggests the tritons and deuterons are not in equilibrium with 


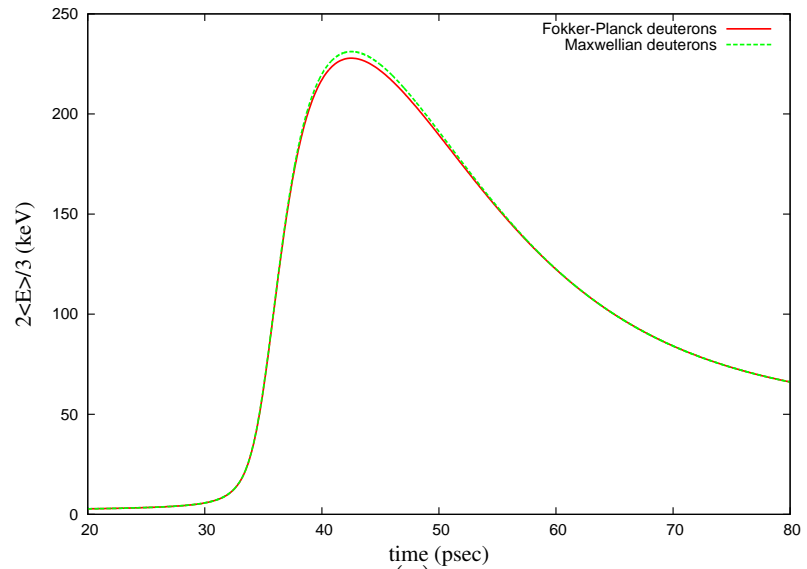

(a)

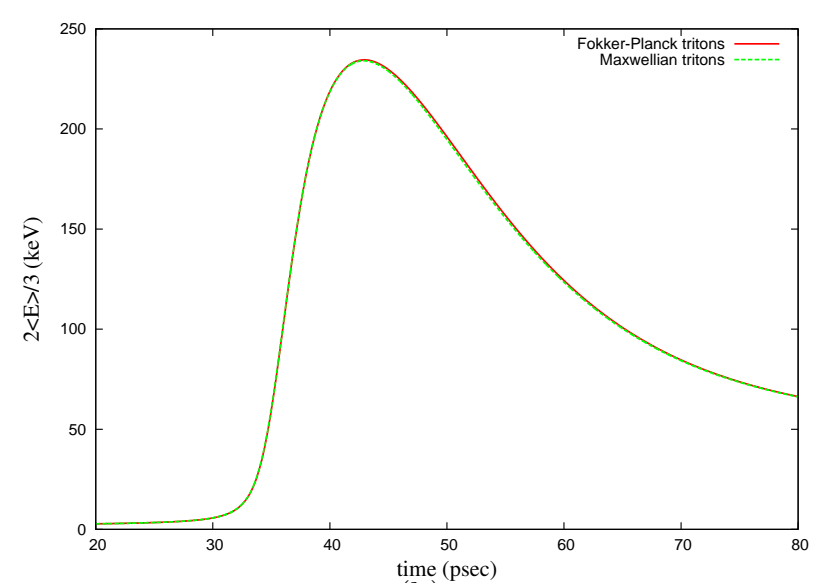

(b)

FIG. 8: Comparison of mean energies between full Fokker-Planck run and run where Maxwellian distributions are used after every timestep. Panel (a) shows comparison for deuteron mean energies, (b) shows comparison for triton mean energies. Note that for runs using Maxwellian distributions, the ordinate is equivalent to temperature.

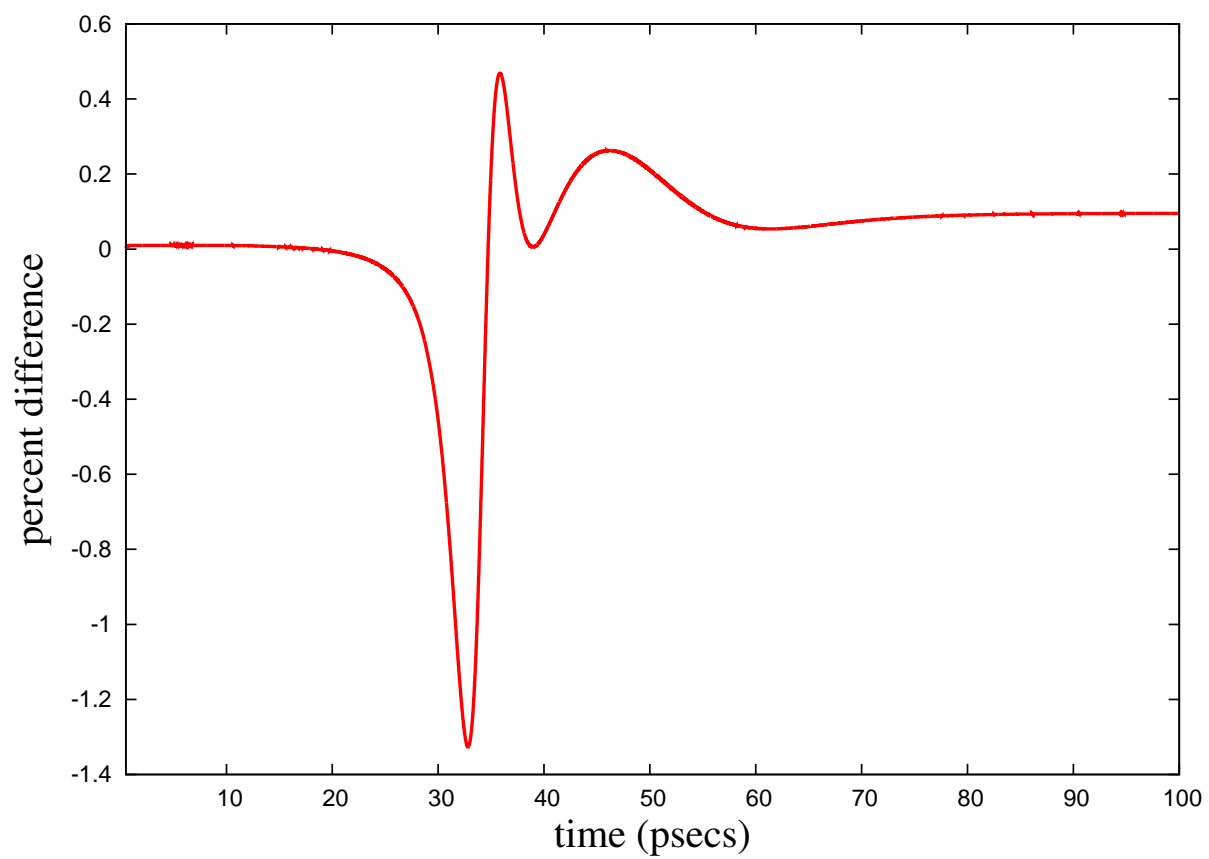

FIG. 9: Relative difference between Fokker-Planck DT fusion rates and thermal DT fusion rates. each other during these times. 


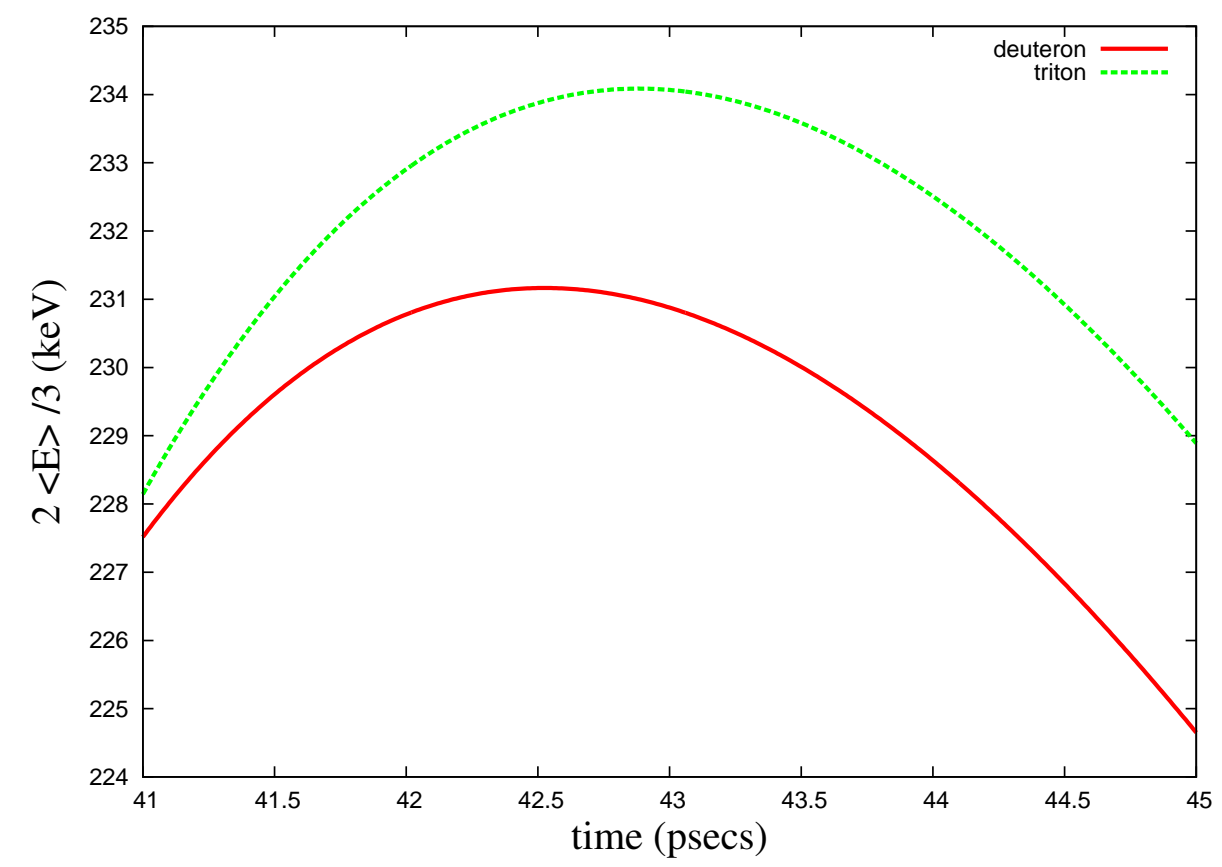

FIG. 10: Mean energies of tritons and deuterons around their peak values.

\section{Fusion product distributions}

Figure 11 shows the fusion products' energy distributions (alpha, proton, and Helium-3), normalized to their respective number densities, at various times during the burn simulation. As was the case with the tritium distribution shown in fig. 5, there are obvious non-equilibrium components to the high-energy regions of the distributions, whereas the low-energy regions are clearly Maxwellian. We again use Eqs. 16 and 17 to extract a bulk thermal background temperature $T_{b g}$ and the number density of particles in equilibrium $N_{\text {eq }}$, respectively. In fig. 12 we plot the background temperatures of the fusion products, as well as the temperatures of the deuterium and tritim ions for comparison. Note that the temperatures are nearly equal to each other, the largest difference occuring during peak burn. We show in fig. 13(a) the percentage of fusion particles not in equilibrium (compare to fig. 6). The percentages are much larger then their deuterium/tritium counterparts, particularly during the lighting stage. Figure. 13(b) shows the excess kurtosis as a function of time (compare to fig. 7). Again these results are much larger than their deuterium/tritium counterparts. 


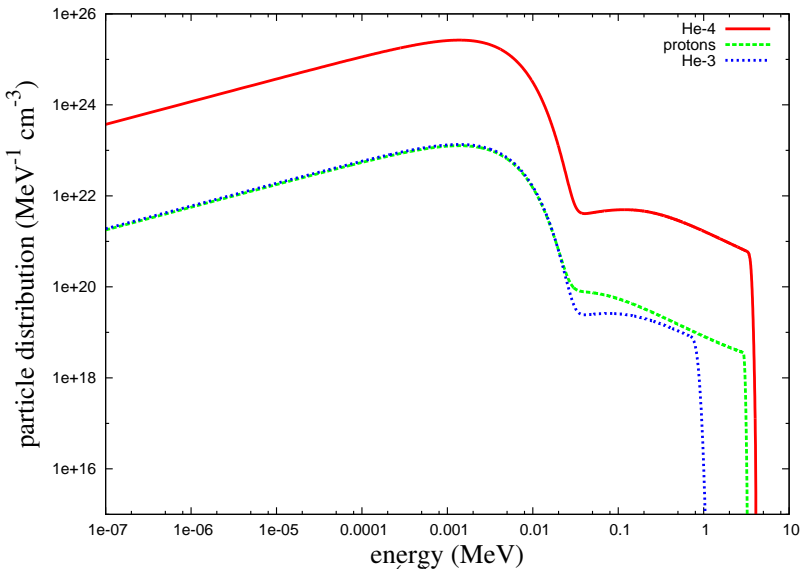

(a)

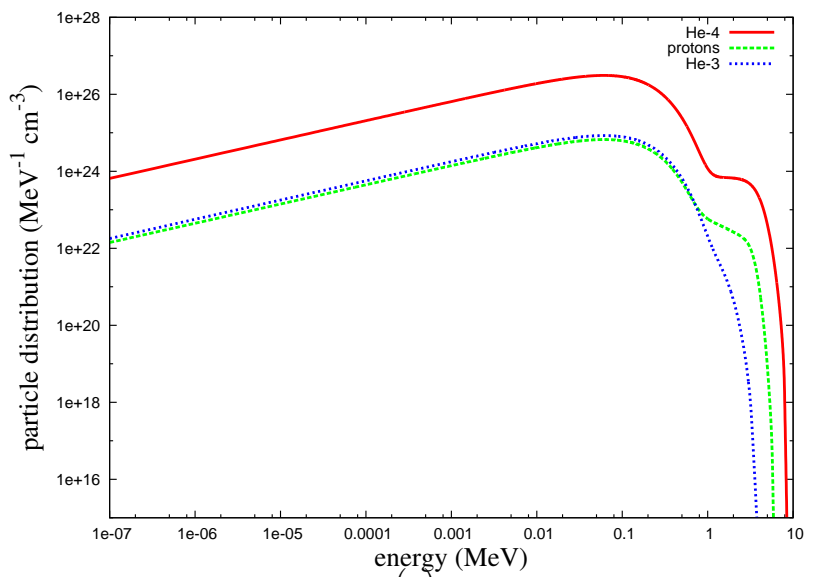

(c)

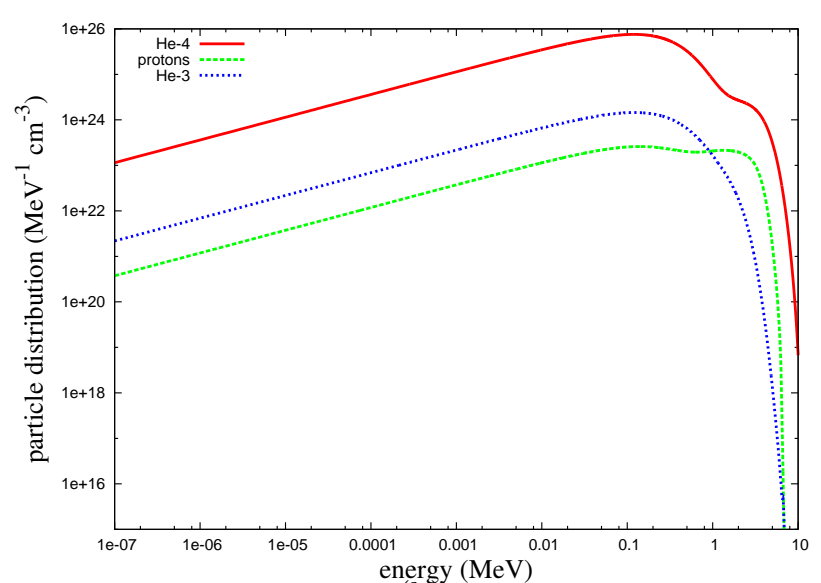

(b)

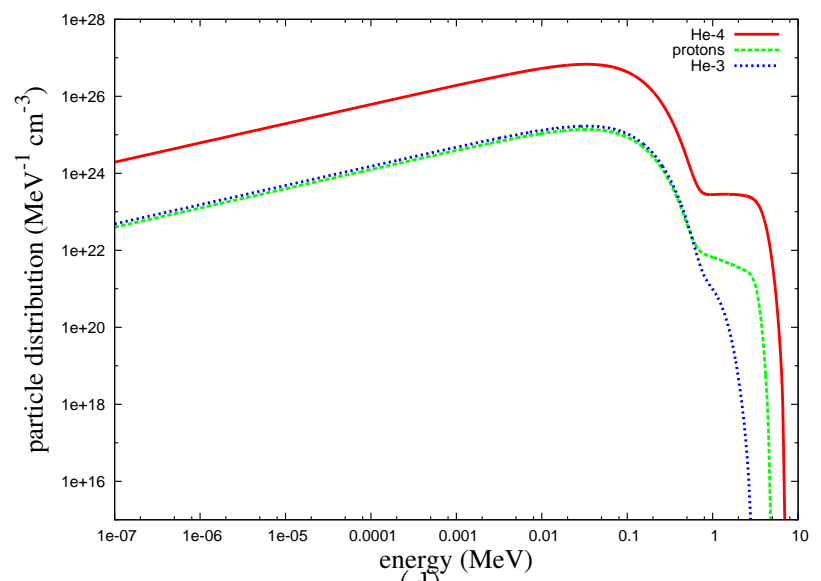

(d)

FIG. 11: Fusion products' energy distributions at various times. Panel (a) shows distributions at $\mathrm{t}=20$ psecs, (b) $\mathrm{t}=40$ psecs, $(\mathrm{c}) \mathrm{t}=60$ psecs, and (d) $\mathrm{t}=80.0$ psecs.

\section{Photon distributions}

In fig. 14 we show results comparing full Fokker-Planck runs (solid red) to runs where the photon distribution is enforced to be Planckian after every timestep (dashed green). Initial conditions are the same as the previous examples. In panel (c) of this figure we have used a temperature label for the ordinate since in previous sections we have shown that deuterons are essentially in equilibrium throughout our simulations.

As opposed to previous deuterium/tritium results, enforcing Planckian photon distributions can dramatically quench fusion burning and alter the evolution of our burn simulations. Figure 14(d) shows the reasoning behind this. During the lighting phase, the photon number density coming from a blackbody distribution grows more quickly than its non-Plancking counterpart. With more photons available to absorb energy coming from fusion, the lighting 


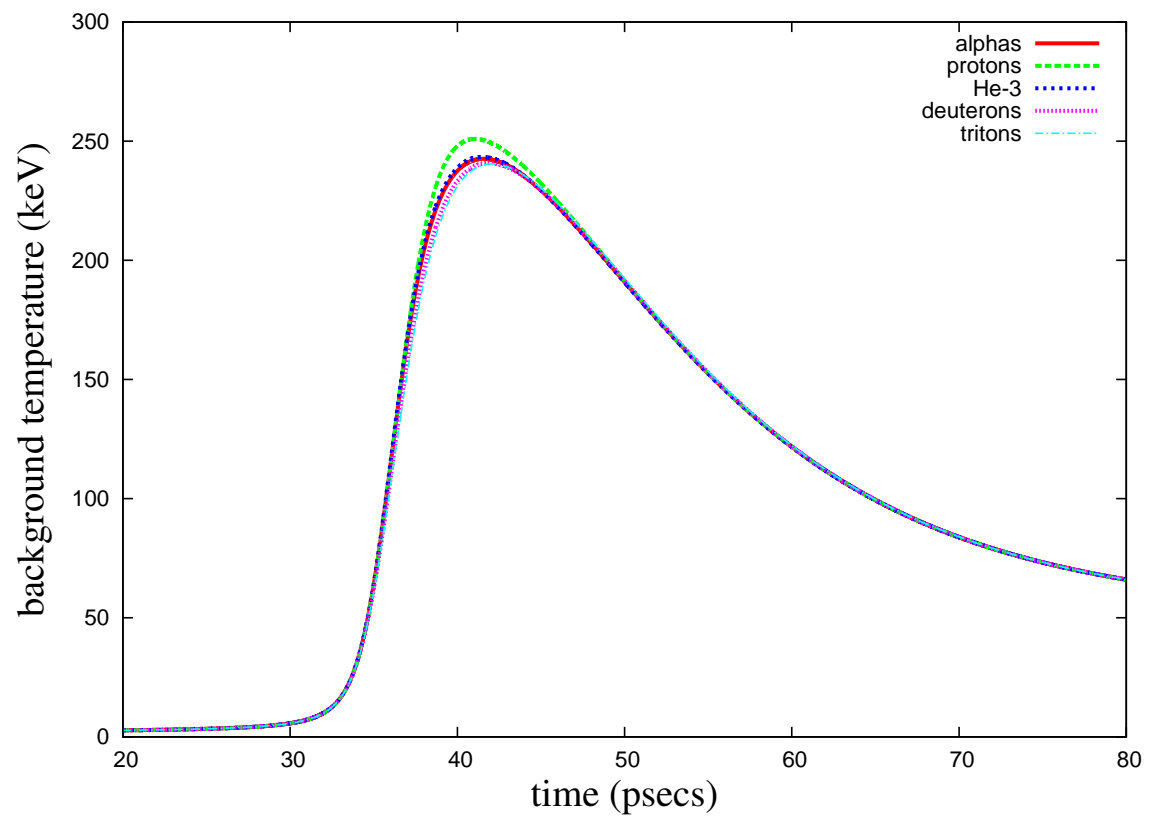

FIG. 12: Background temperatures derived from fitting Maxwell-Boltzmann distributions to lowenergy distributions shown in fig. 11. Also shown are deuteron and triton temperatures for comparision.

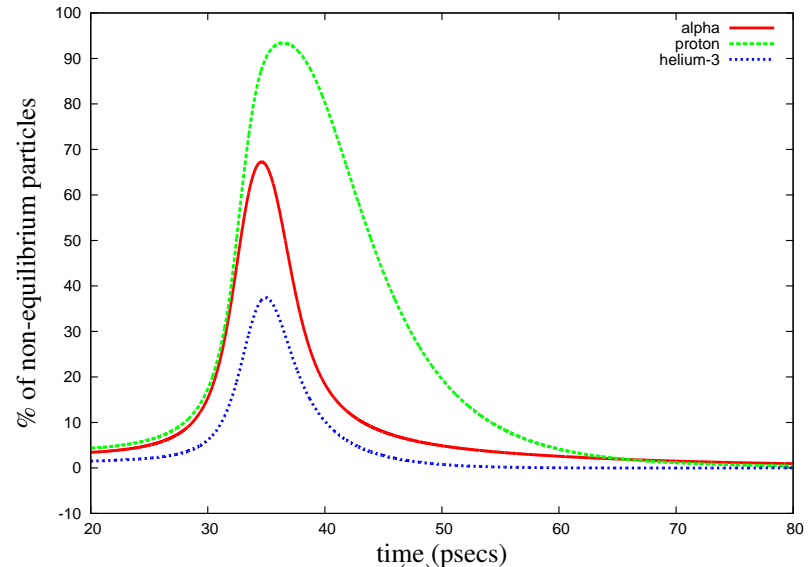

(a)

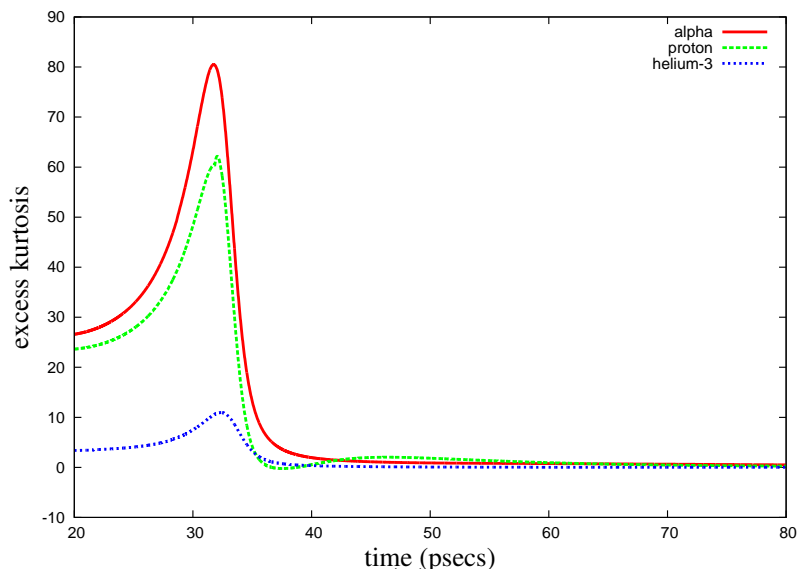

(b)

FIG. 13: Percentage of fusion products not in equilibrium (a) and excess kurtosis of fusion products (b) as a function of time.

phase becomes quenched. In essence, the 'specific heat' of the photon bath is increased. 


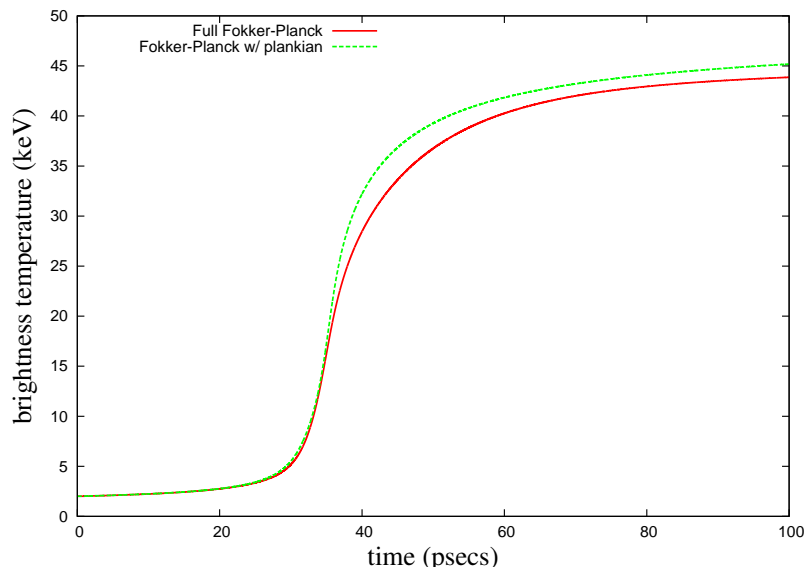

(a)

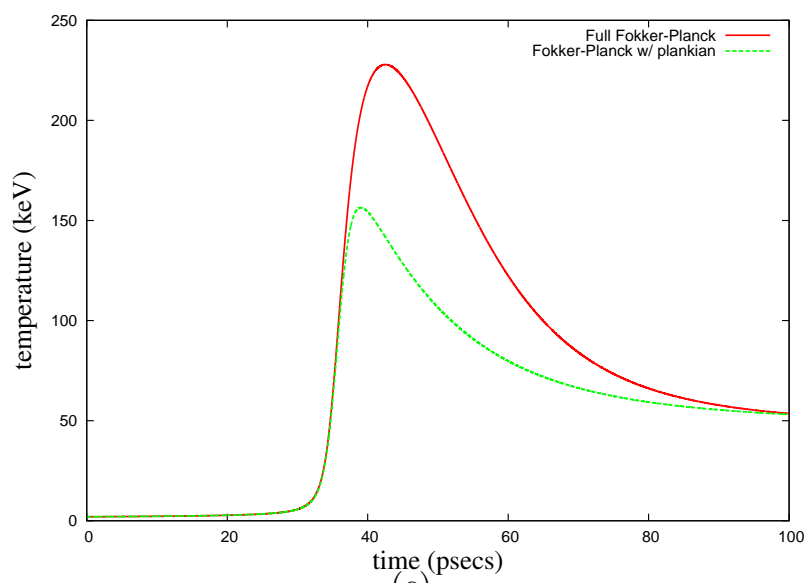

(c)

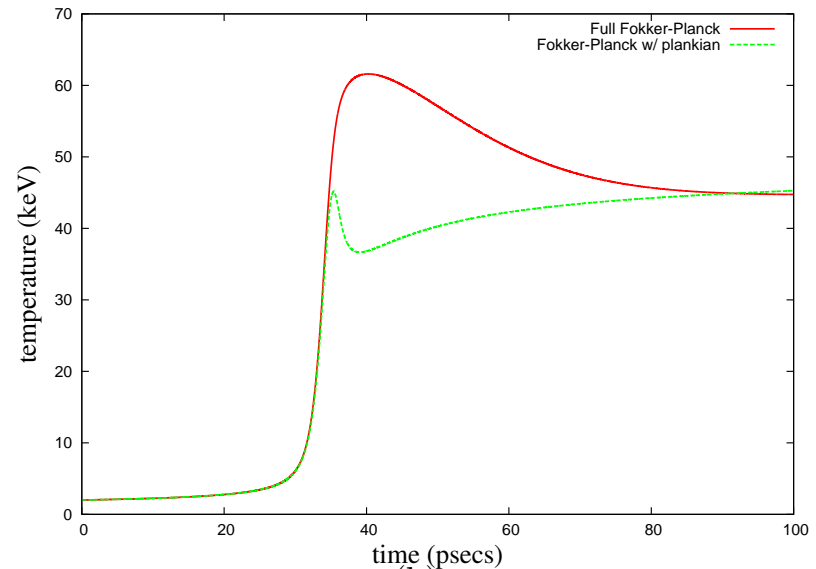

(b)

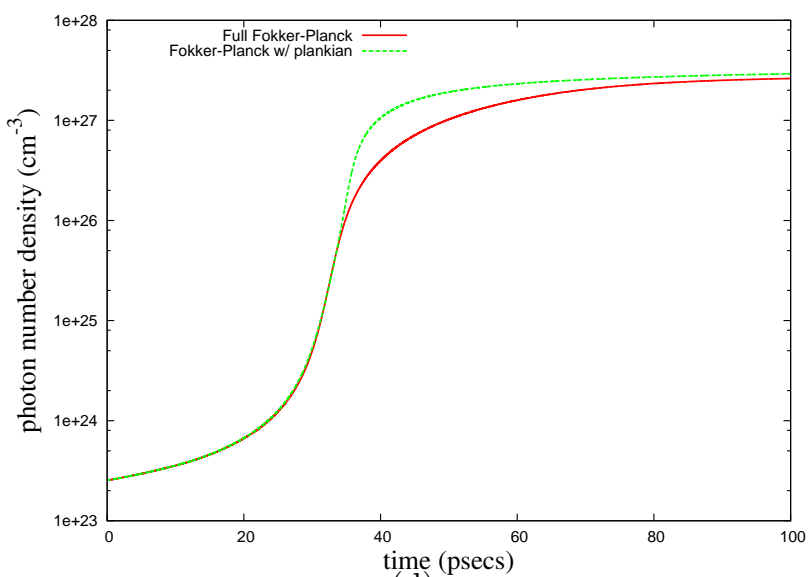

(d)

FIG. 14: Results comparing full Fokker-Planck runs (red) and Fokker-Planck runs with Planckian photon distributions enforced after every timestep (green). Panel (a) shows difference between photon effective temperatures, (b) shows difference in electron temperatures, (c) shows difference in deuteron temperatures, and (d) shows difference in photon number densities.

\section{Other initial conditions}

In fig. 15 we show the electron and ion mean energies of other simulations using different initial conditions, as labeled in the figures. It is clear from these runs that for a given initial density of deuterium and tritium, the largest effect due to different initial temperatures is to delay or advance the onset of runaway burn. Otherwise, the burn profile remains the same. Increasing the initial densities, while keeping the same initial temperatures, advances the 'lighting' stage and augments the peak ion energies. As a rough rule of thumb, we have found that increasing the initial density by an order of magnitude reduces the time for the onset of runaway burn by an order of magnitude. 


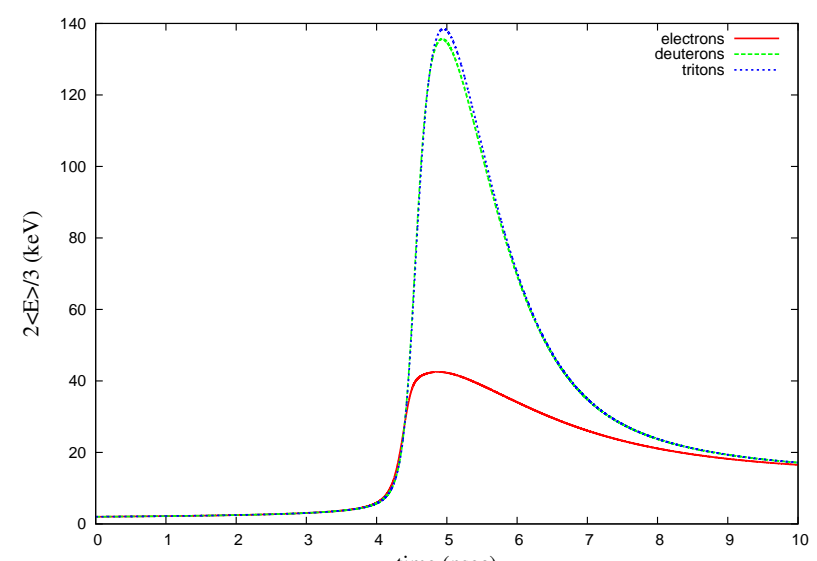

(a)

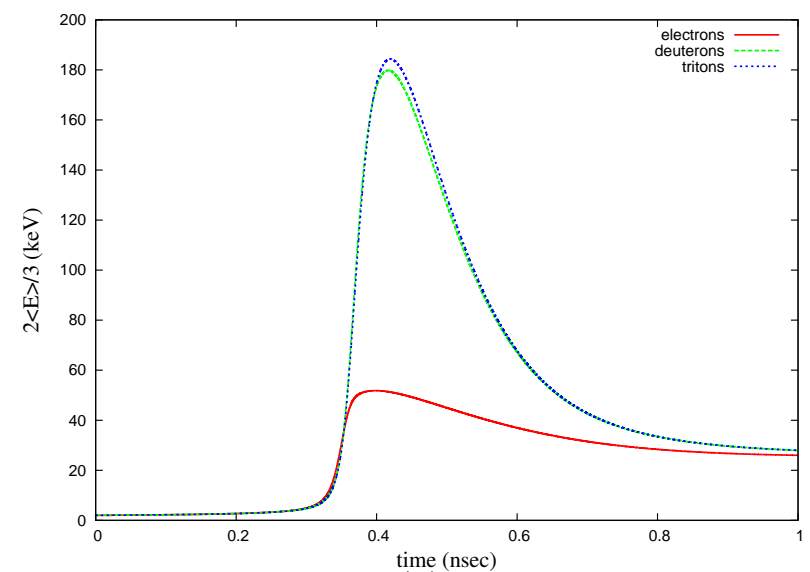

(d)

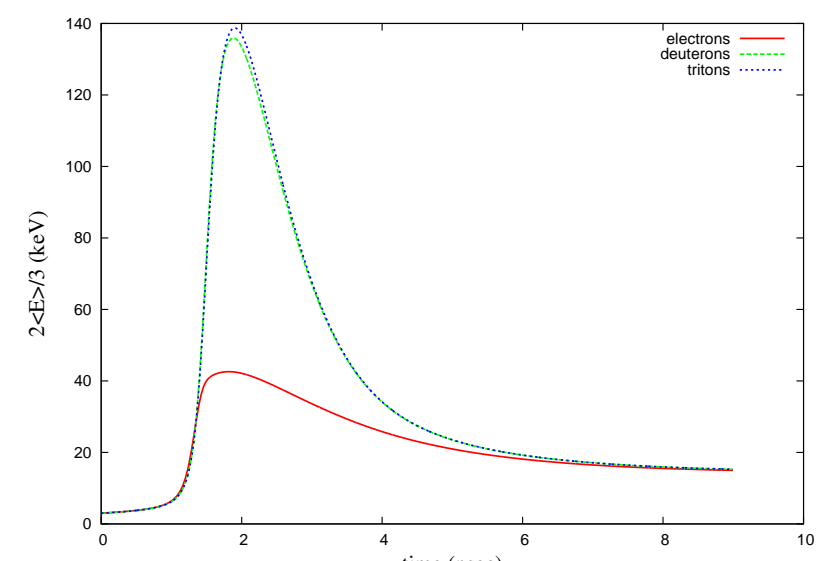

(b)

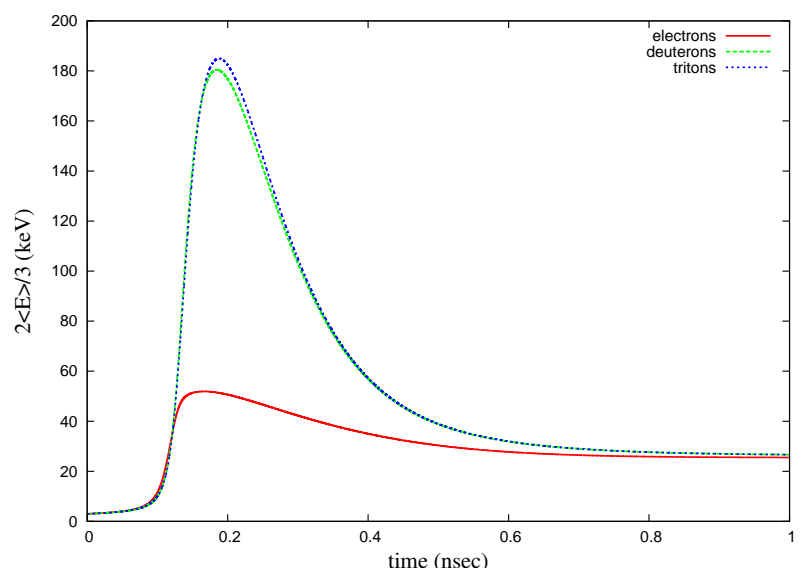

(e)

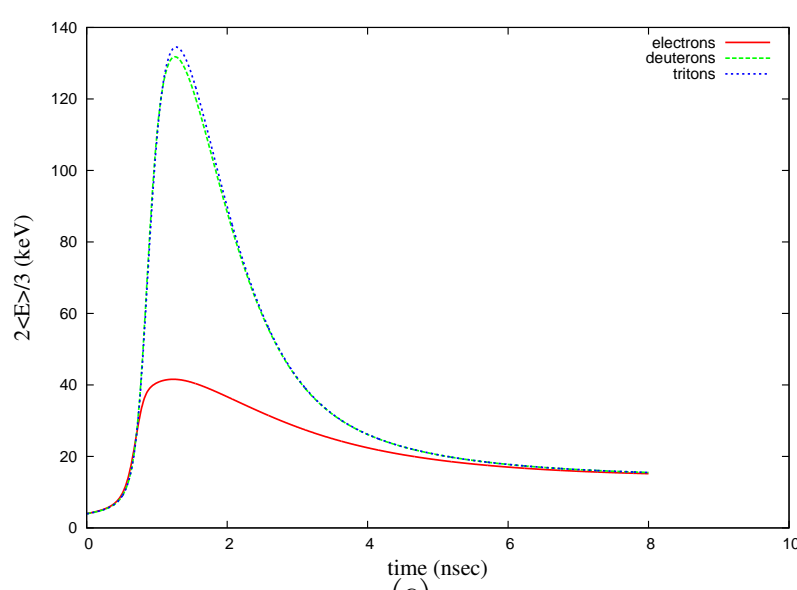

(c)

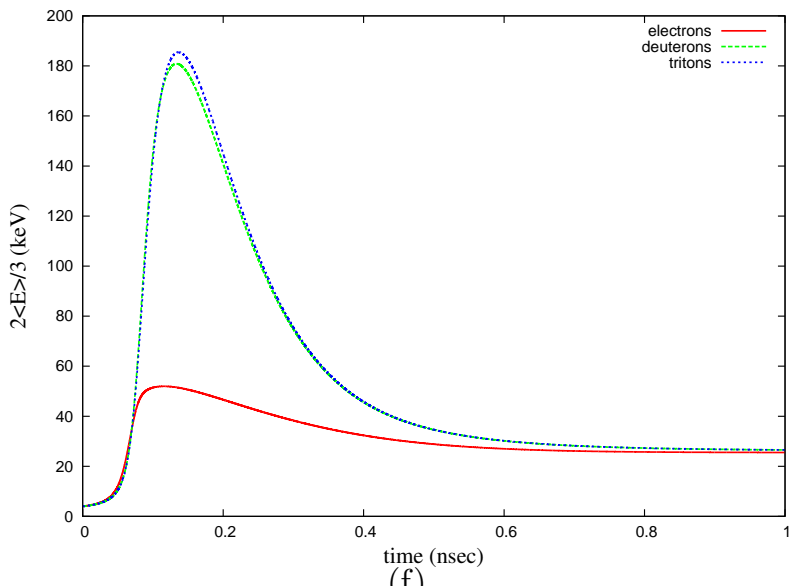

(f)

FIG. 15: Burn profiles for runs with different initial conditions. Panels (a), (b), and (c) had $10 \mathrm{gm} / \mathrm{cm}^{3}$ initial concentrations of DT fuel, whereas (d), (e), and (f) had $100 \mathrm{gm} / \mathrm{cm}^{3}$. Panels (a) and (d) had all species initially held at $\mathrm{kT}=2 \mathrm{keV}$, (b) and (e) held at kT = $3 \mathrm{keV}$, and (c) and (f) held at $\mathrm{kT}=4 \mathrm{keV}$. 
We have performed detailed analysis of these runs similar to those described in the above sections. Our conclusions remain the same. Namely, non-thermal components of triton and deuteron distributions have little, if any, effects in the evolution of burning plasmas, whereas non-Planckian photon distributions play a large role in the evolution of burning plasmas.

\section{AFFECT OF XENON DOPANT}

In fig. 16 we show how the burn evolution is altered when the presence of a high- $Z$ dopant (in this case Xenon) is included in the gas. Initial conditions have all particles at $\mathrm{kT}=2 \mathrm{keV}$ and concentration of D-T fuel at $10 \mathrm{~g} / \mathrm{cm}^{3}$. Scenario $A$ has no Xenon dopant, $B$ has Xenon dopant at $10^{-4}$ the amount of initial deuterium, $C$ has Xenon dopant at $10^{-3}$ the amount of initial deuterium, and $D$ is $10^{-2}$ the amount of initial deuterium. The Xenon dopant remains in equilibrium with the deuterons (and tritons) throughout the burn process, as is evident by the equal temperatures seen in fig. 16(b) and (d). Clearly the effects of high-Z dopants tends to quench and delay the burn, as more fusion energy is absorbed by the dopants and less energy is available to drive the fusion reactants.

We note that in these calculations we treat the Xenons as fully ionized, and thus only 'freefree' Compton scattering processes occur. This is not an accurate description of the entire physical processes, since the Xenons will not be entirely ionized; rather, their ionization will be a function of temperature. However, our results should still be qualitatively correct.

\section{CONCLUSION}

To answer the question of whether fusing plasmas can produce non-equilibrium distributions, we have developed a Fokker-Planck algorithm that incorporates thermo-nuclear fusion. Our findings suggest that deuterium and tritium ions remain very close to equilibrium with each other even during runaway burn. This is not the case for fusion products. They retain a significant non-thermal part to their distributions. The distribution of these fusion products can be split into a thermal 'bulk' part, described by a bulk temperature, and a remaining non-thermal part. We find that the 'bulk' ions essentially remain in equilibrium with the reacting ions.

The radiation field does not retain its Planckian distribution during runway burn and rep- 


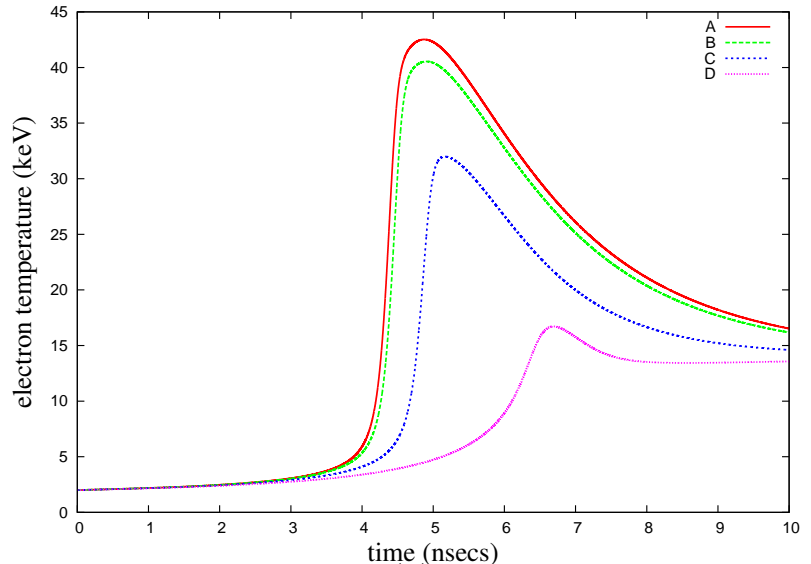

(a)

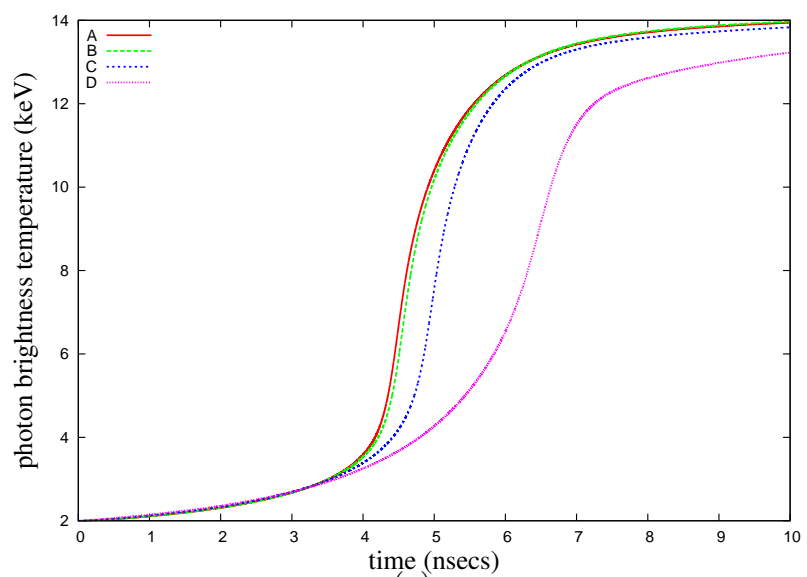

(c)

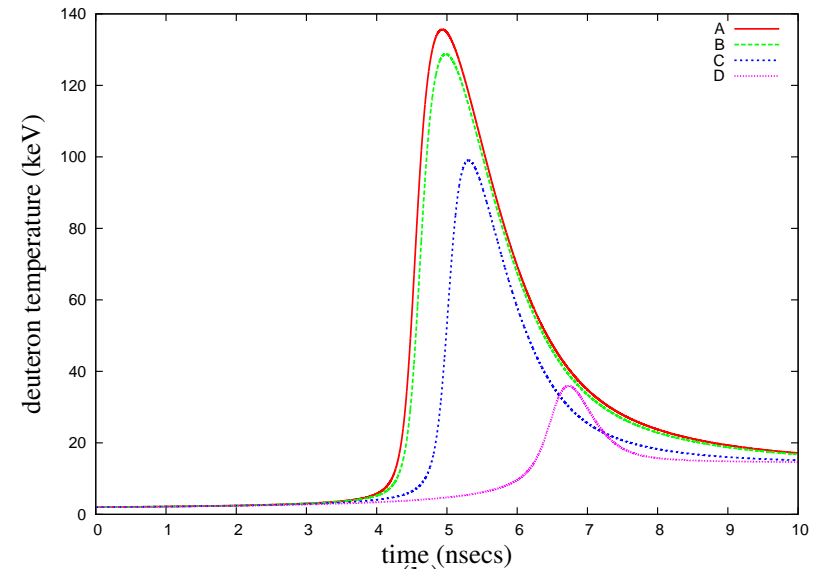

(b)

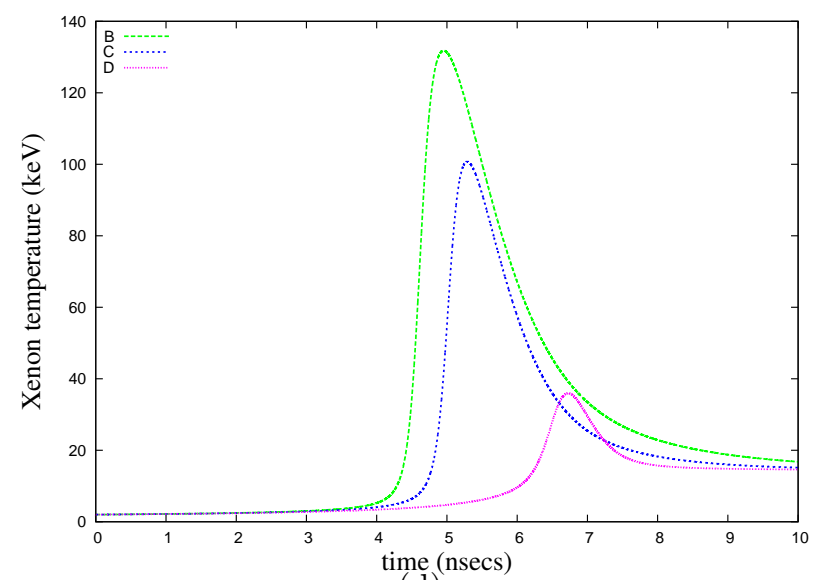

(d)

FIG. 16: Effect of Xenon dopant on electron,deuteron, photon, and Xenon temperatures. Line $A$ has no dopant, $B$ has dopant at $10^{-4}$ the amount of initial deuterium, $C 10^{-3}$, and $D 10^{-2}$.

resents the largest non-equilibrium component of the burning plasma. Enforcing Planckian distributions on the radiation after every timestep also drastically alters the burn evolution by effectively quenching the burn and delaying the lighting stage.

We have also simulated the effects of dopants, or high-Z material, on the evolution of the burning plasma by placing fractional amounts in the plasma. As expected, the dopants delay the onset of runaway burn, as well as quenches the burn. The dopants remain in equilibrium with the reacting ions, however.

Our results show that the depletion of the deuteron and triton distributions around the Gamow peak due to fusion alone is not sufficient to drive non-equilibrium physics for the reacting ions. Because our investigations were taylored to ICF environments where neutron areal densities are large compared to areal densities of ICF targets, our initial studies did 
not track the fusion neutrons and, in particular, did not account for any energy depostion due to plasma interactions with these neutrons. Further, we did not account for large-angle Coulomb scattering or elastic reaction-in-flight processes with ions produced through fusion. These processes could, in principle, induce non-thermal effects. We plan to include these physical processes into our Fokker-Planck algorithm for future studies.

\section{Acknowledgments}

We thank Des Pilkington for useful comments and insightful critiques of this manuscript. This work was performed under the auspices of the U.S. Department of Energy by Lawrence Livermore National Laboratory under Contract DE-AC52-07NA27344.

[1] S. Atzeni and J. M. ter Vehn, The physics of inertial fusion (Clarendon Press, 2004).

[2] J. Lindl, Physics of Plasmas 2, 3933 (1995).

[3] G. Dimonte and J. Daligault, Physical Review Letters 101, 135001 (2008).

[4] L. Benedict, J. Glosli, and D. R. et al., Physical Review Letters 102, 205004 (2009).

[5] L. Spitzer, Physics of Fully Ionized Gases (Interscience, 1967).

[6] E. Lifshitz and L. Piataevskii, Physical Kinetics (Butterworth and Heinemann, 1979).

[7] J. C. Whitney, Journal of Computational Physics 6, 483 (1970).

[8] J. Chang and G. Cooper, Journal of Computational Physics 6, 1 (1970).

[9] E. Epperlein, Journal of Computational Physics 112, 291 (1994).

[10] D. O. Gericke, M. S. Murillo, and M. Schlanges, Phys. Rev. E 65, 036418 (2002).

[11] A. Langdon, CECAM Report of Workshop on The Flux Limiter and Heat Flow Instabilities in Laser-Fusion Plasmas (1981).

[12] A. Kompaneets, Sov. Phys., JETP 4, 730 (1957).

[13] G. Rybicki and A. Lightman, Radiative Processes in Astrophysics (Wiley Interscience, 1979).

[14] M. Baring, Mon. Not. R. astr. Soc. 228, 681 (1987).

[15] D. Shirk, Tech. Rep. LA-14297, Los Alamos National Laboratory (2006).

[16] Under the current approximations discussed in previous sections 